\title{
INTERPOLATION BY ASYMMETRIC, TWO-DIMENSIONAL CUBIC CONVOLUTION
}

\author{
Jiazheng Shi and Stephen E. Reichenbach \\ Computer Science and Engineering Department \\ University of Nebraska - Lincoln, Lincoln NE 68588-0115 USA
}

\begin{abstract}
This paper formulates a two-dimensional, piecewise-cubic interpolator that accounts for non-separable and rotationally asymmetric scene statistics. The resulting kernel has five parameters and is designated 2D-5PCC. As a low-order polynomial with small spatial support, the kernel is easy to implement and efficient to apply. A closed-form solution, developed in this paper, yields the optimal parameter values for scene ensembles characterized by autocorrelation or power spectrum. Experiments indicate that 2D-5PCC yields higher fidelity interpolations than several other popular methods.
\end{abstract}

\section{INTRODUCTION}

Image interpolation is the process of defining a spatially continuous image from a set of discrete samples. Interpolation is fundamental to many digital image processing applications, particularly in operations requiring image resampling, such as image warping, correction for geometric distortions, and image registration. Interpolation commonly is implemented by convolving an image with a small kernel for the weighting function. Popular methods of interpolation include nearest neighbor interpolation, bi-linear interpolation, cubic B-spline interpolation, and piecewise-cubic convolution (PCC) [1]. PCC has been used for image interpolation since the 1970's and provides a good compromise between computational complexity and interpolation accuracy [2]. Cubic convolution can be parameterized and then optimized either for general performance characteristics or for optimal fidelity over an image ensemble with specific characteristics [3, 4].

This paper formulates a PCC interpolator that accounts for non-separable and rotationally asymmetric scene statistics. This kernel is the most general two-dimensional, biaxial symmetric, piecewise-cubic interpolator defined on [$2,2] \times[-2,2]$ with constraints for continuity and smoothness. This paper also develops a closed-form solution for the optimal parameters subject to any set of constraints.

This work was supported by the National Science Foundation, Digital Government Program, Award EIA-0091430. Contact information: email: [jshi|reich]@cse.unl.edu, phone: 1.402.472.2401, fax: 1.402.472.7767.

\section{FORMULATION OF 2D-5PCC}

Interpolation aims to recreate a continuous function from discrete samples. Let $s(x, y),(x, y \in \mathbb{R})$, be a continuous two-dimensional scene and $s[m, n],(m, n \in 0, \pm 1, \pm 2, \ldots)$, be an image consisting of uniformly spaced discrete samples from the scene. Many popular image interpolation methods, including nearest-neighbor, bi-linear, cubic convolution, and cubic-spline interpolation implement interpolation by convolution of the image $s[m, n]$ with a continuous two dimensional kernel $f(x, y),(x, y \in \mathbb{R})$ :

$$
r(x, y)=\sum_{m=-\infty}^{+\infty} \sum_{n=-\infty}^{+\infty} s[m, n] f(x-m, y-n) .
$$

In the Fourier frequency domain:

$$
\hat{r}(u, v)=\hat{f}(u, v) \sum_{\mu=-\infty}^{+\infty} \sum_{\nu=-\infty}^{+\infty} \hat{s}(u-\mu, v-\nu),
$$

where $\hat{r}(u, v), \hat{f}(u, v)$, and $\hat{s}(u, v)$ are the Fourier transforms of the interpolated result $r(x, y)$, the interpolation kernel $f(x, y)$, and the scene $s(x, y)$ respectively.

Traditionally, PCC is implemented by separable convolution with a small one-dimensional kernel consisting of one parameter and piecewise-cubic polynomials (designated 1D1PCC). This method can be generalized to two dimensions in a nonseparable fashion. With constraints for flat-field interpolation, biaxial and diagonal symmetry, continuity, and smoothness, the kernel can be reduced to a two-dimensional function of three parameters (2D-3PCC) [5]. However, many scenes do not have rotational symmetry. Relaxing the constraint on $90^{\circ}$ rotational symmetry, yields a five parameter kernel, designated 2D-5PCC:

$$
\begin{aligned}
f(x, y)= & a_{33} f_{33}(x, y)+a_{32} f_{32}(x, y)+a_{23} f_{23}(x, y) \\
& +a_{30} f_{30}(x, y)+a_{03} f_{03}(x, y)+f_{00}(x, y),
\end{aligned}
$$

where $a_{33}, a_{32}, a_{23}, a_{30}$, and $a_{03}$ are the five parameters and $f_{33}, f_{32}, f_{23}, f_{30}, f_{03}$, and $f_{00}$ are defined in Fig. 1. Fig. 2 shows the spatial-domain point spread functions (PSFs) of the parametric components. The components $f_{30}(x, y)$ and $f_{03}(x, y)$ provide capacity for directional edge enhancement. 


$$
\begin{aligned}
& f_{33}(x, y)=\left\{\begin{array}{l}
x^{3} y^{3}-x^{2} y^{2} \\
(x-2)^{2}\left(5 x y^{3}-4 x y^{2}-4 y^{3}+3 y^{2}\right) \\
(x-2)^{2}(y-2)^{2}(9 x y-8 x-8 y+7) \\
(y-2)^{2}\left(5 x^{3} y-4 x^{2} y-4 x^{3}+3 x^{2}\right)
\end{array}\right. \\
& f_{32}(x, y)=\left\{\begin{array}{l}
x^{3} y^{2}-x^{2} y^{2} \\
(x-2)^{2}\left(x y^{2}-y^{2}\right) \\
(y-2)^{2}(x-2)^{2}(4 x y-4 y-3 x+3) \\
(y-2)^{2}\left(4 x^{3} y-4 x^{2} y-3 x^{3}+3 x^{2}\right)
\end{array}\right. \\
& f_{23}(x, y)=\left\{\begin{array}{l}
x^{2} y^{3}-x^{2} y^{2} \\
(x-2)^{2}\left(4 x y^{3}-4 x y^{2}-3 y^{3}+3 y^{2}\right) \\
(y-2)^{2}(x-2)^{2}(4 x y-4 x-3 y+3) \\
(y-2)^{2}\left(x^{2} y-x^{2}\right)
\end{array}\right. \\
& f_{30}(x, y)=\left\{\begin{array}{l}
x^{3}-x^{2} \\
(x-2)^{2}(x-1) \\
(x-2)^{2}(y-2)^{2}(2 x y-2 y-x+1) \\
(y-2)^{2}\left(2 x^{3} y-2 x^{2} y-x^{3}+x^{2}\right)
\end{array}\right. \\
& f_{03}(x, y)=\left\{\begin{array}{l}
y^{3}-y^{2} \\
(x-2)^{2}\left(2 x y^{3}-2 x y^{2}-y^{3}+y^{2}\right) \\
(y-2)^{2}(x-2)^{2}(2 x y-2 x-y+1) \\
(y-2)^{2}(y-1)
\end{array}\right. \\
& f_{00}(x, y)=\left\{\begin{array}{l}
x^{2} y^{2}-x^{2}-y^{2}+1 \\
(x-2)^{2}\left(2 x y^{2}-2 y^{2}-2 x+2\right) \\
(y-2)^{2}(x-2)^{2}(4 x y-4 y-4 x+4) \\
(y-2)^{2}\left(2 x^{2} y-2 x^{2}-2 y+2\right)
\end{array}\right.
\end{aligned}
$$

$0 \leq x \leq 1,0 \leq y \leq 1$

$1<x \leq 2,0 \leq y \leq 1$

$1<x \leq 2,1<y \leq 2$

$0 \leq x \leq 1,1<y \leq 2$

$0 \leq x \leq 1,0 \leq y \leq 1$

$1<x \leq 2,0 \leq y \leq 1$

$1<x \leq 2,1<y \leq 2$

$0 \leq x \leq 1,1<y \leq 2$

$0 \leq x \leq 1,0 \leq y \leq 1$

$1<x \leq 2,0 \leq y \leq 1$

$1<x \leq 2,1<y \leq 2$

$0 \leq x \leq 1,1<y \leq 2$

$0 \leq x \leq 1,0 \leq y \leq 1$

$1<x \leq 2,0 \leq y \leq 1$

$1<x \leq 2,1<y \leq 2$

$0 \leq x \leq 1,1<y \leq 2$

$0 \leq x \leq 1,0 \leq y \leq 1$

$1<x \leq 2,0 \leq y \leq 1$

$1<x \leq 2,1<y \leq 2$

$0 \leq x \leq 1,1<y \leq 2$

$0 \leq x \leq 1,0 \leq y \leq 1$

$1<x \leq 2,0 \leq y \leq 1$

$1<x \leq 2,1<y \leq 2$

$0 \leq x \leq 1,1<y \leq 2$.

Fig. 1. Component equations for 2D-5PCC in Eq. 3.

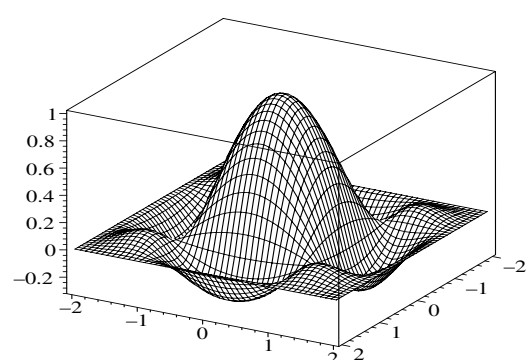

A. $f_{00}(x, y)$

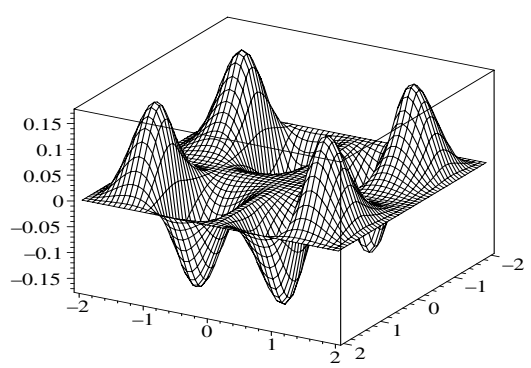

D. $f_{23}(x, y)$

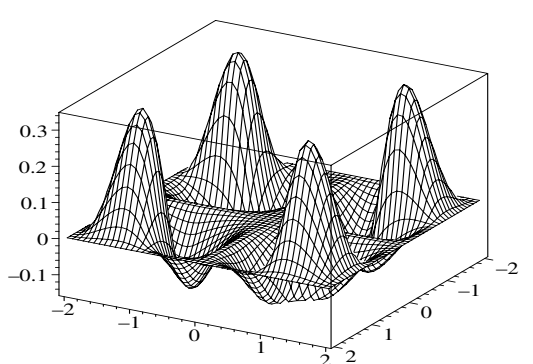

B. $f_{33}(x, y)$

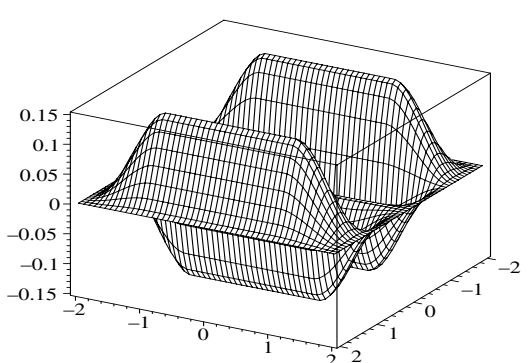

E. $f_{30}(x, y)$

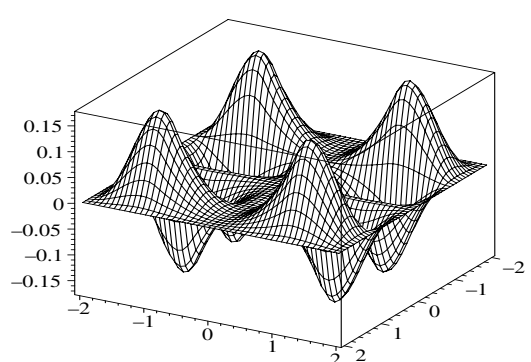

C. $f_{32}(x, y)$

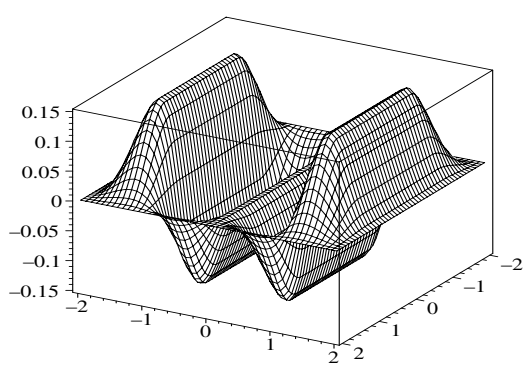

F. $f_{03}(x, y)$

Fig. 2. Component plots for 2D-5PCC in Eq. 3. 


\section{OPTIMAL PARAMETERIZATION}

This section develops a closed-form solution for the optimal parameters for 2D-5PCC, based on an analysis of the expected mean square error (MSE) $\epsilon^{2}$ for interpolation of an ensemble of scenes characterized by autocorrelation or power spectrum. By Rayleigh's Theorem, the expected MSE of a system for an ensemble of scenes can be analyzed in either the spatial or frequency domain:

$$
\begin{aligned}
\epsilon^{2} & =E\left\{\iint|r(x, y)-s(x, y)|^{2} d x d y\right\} \\
& =E\left\{\iint|\hat{r}(u, v)-\hat{s}(u, v)|^{2} d u d v\right\} .
\end{aligned}
$$

If co-aliased components of the sampled scene are uncorrelated [6], the expected MSE can be expressed in terms of the scene power spectrum and the interpolation kernel:

$$
\begin{aligned}
\epsilon^{2}= & \iint\left(\hat{\Phi}_{s}(u, v)-2 \hat{f}(u, v) \hat{\Phi}_{s}(u, v)\right. \\
& \left.+|\hat{f}(u, v)|^{2} \hat{\Phi}_{p}(u, v)\right) d u d v,
\end{aligned}
$$

where $\hat{\Phi}_{s}(u, v)$ is the scene power spectrum and

$$
\hat{\Phi}_{p}(u, v)=\sum_{\mu} \sum_{\nu} \hat{\Phi}_{s}(u-\mu, v-\nu),
$$

is the image power spectrum (with aliasing). Without loss of generality, scenes are normalized so that the mean and variance are zero and one respectively.

The MSE $\epsilon^{2}$ is a quadratic function of the parameters $\left(a_{33}, a_{32}, a_{23}, a_{30}\right.$ and $\left.a_{03}\right)$, so the optimal values can be derived by substituting the Fourier transform of the kernel from Eq. 3 into Eq. 5, computing the partial derivatives of $\epsilon^{2}$ with respect to the parameters, and solving for simultaneous equality with zero:

$$
\frac{\partial \epsilon^{2}}{\partial a_{33}}=\frac{\partial \epsilon^{2}}{\partial a_{32}}=\frac{\partial \epsilon^{2}}{\partial a_{23}}=\frac{\partial \epsilon^{2}}{\partial a_{30}}=\frac{\partial \epsilon^{2}}{\partial a_{03}}=0 .
$$

This yields five equations for the optimal parameter values:

$$
\begin{aligned}
& \iint \hat{g}_{i}(u, v)\left(\hat{\Phi}_{s}(u, v)-\hat{f}_{00}(u, v) \hat{\Phi}_{p}(u, v)\right) d u d v \\
& =\iint \hat{g}_{i}(u, v)\left(\hat{f}(u, v)-\hat{f}_{00}(u, v)\right) \hat{\Phi}_{p}(u, v) d u d v
\end{aligned}
$$

for $\hat{g}_{0}=\hat{f}_{33}, \hat{g}_{1}=\hat{f}_{32}, \hat{g}_{2}=\hat{f}_{23}, \hat{g}_{3}=\hat{f}_{30}$, and $\hat{g}_{4}=\hat{f}_{03}$. With matrix notation, Eq. 8 can be written as $K=H A$ :

$$
\left[\begin{array}{l}
k_{0} \\
k_{1} \\
k_{2} \\
k_{3} \\
k_{4}
\end{array}\right]=\left[\begin{array}{lllll}
h_{00} & h_{01} & h_{02} & h_{03} & h_{04} \\
h_{10} & h_{11} & h_{12} & h_{13} & h_{14} \\
h_{20} & h_{21} & h_{22} & h_{23} & h_{24} \\
h_{30} & h_{31} & h_{32} & h_{33} & h_{34} \\
h_{40} & h_{41} & h_{42} & h_{43} & h_{44}
\end{array}\right]\left[\begin{array}{l}
a_{33} \\
a_{32} \\
a_{23} \\
a_{30} \\
a_{03}
\end{array}\right],
$$

where:

$$
\begin{aligned}
k_{i} & =\iint \hat{g}_{i}(u, v)\left(\hat{\Phi}_{s}(u, v)-\hat{f}_{00}(u, v) \hat{\Phi}_{p}(u, v)\right) d u d v \\
h_{i j} & =\iint \hat{g}_{i}(u, v) \hat{g}_{j}(u, v) \hat{\Phi}_{p}(u, v) d u d v .
\end{aligned}
$$

Then, the optimal parameter values can be solved as:

$$
A=H^{-1} K
$$

if $H$ is well-conditioned.

If the scene statistics have $90^{\circ}$ rotational symmetry, then the optimal values have $a_{32}=a_{23}$ and $a_{30}=a_{03}$ and 2D5PCC reduces to 2D-3PCC with $a_{32}$ parameterizing $f_{32}+$ $f_{23}$ and $a_{30}$ parameterizing $f_{30}+f_{03}$.

\section{EXPERIMENTAL RESULTS}

This section examines the performance of 2D-5PCC in two aspects: visual quality and quantitative MSE. Fig. 3A shows a fence scene [7]. This $256 \times 256$ scene contains more details along- $x$ than along- $y$. The scene is downsampled and then interpolated back to $256 \times 256$ by various algorithms. The images sampled to $64 \times 64$ and then interpolated are shown in Fig. 3(B)-(H). Visually, 2D-5PCC outperforms traditional cubic convolution (1D-1PCC), the $90^{\circ}$ rotationally symmetric nonseparable cubic kernel (2D-3PCC), cubic B-spline interpolation [8], and cubic o-Moms interpolation [9]. Table 1 lists the MSE $\epsilon^{2}$ of various algorithms for the fence scene. In these experiments, the (optimal, approximating) Wiener filter gives the smallest MSE, but 2D5PCC yields smaller MSE than the other methods.

Note that the Wiener filter is a global filter and so is computationally expensive. It is presented only to benchmark optimal fidelity. Regarding the PCC kernels (1D-1PCC, 2D-3PCC, and 2D-5PCC) and the B-spline family (cubic $\mathrm{B}$-spline interpolation and cubic o-Moms interpolation), the former can balance the error budget tradeoff between signal error caused by alternation of scene signal components at frequencies where $\hat{f}(u, v) \neq 1$ and aliasing error caused by interpolation of the aliased components. The balancing is realized by optimizing parameters of the PCC kernels. Aliasing occurs frequently in many imaging applications where anti-aliasing filters can not be applied on physical matter [10]. As the down-sampling rate increases, aliasing becomes more pronounced and so does the difference of MSE between piecewise-cubic convolution and the B-spline family.

\section{CONCLUSIONS}

This paper presents the most general two-dimensional, nonseparable, biaxial-symmetric, piecewise-cubic interpolator 


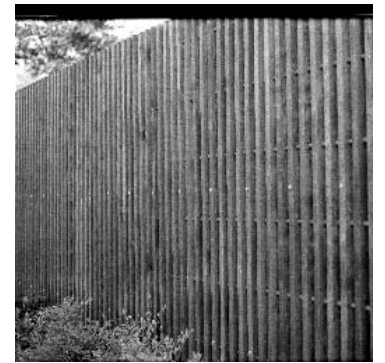

A. Fence scene

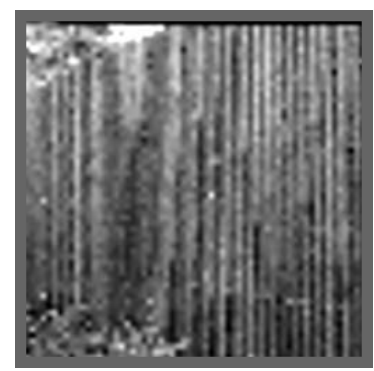

E. 1D-1PCC

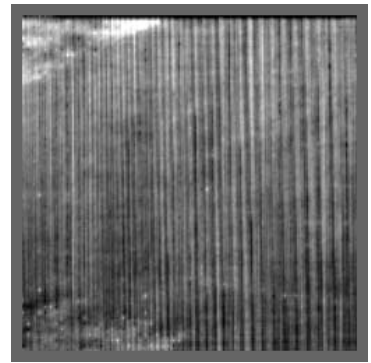

B. Wiener

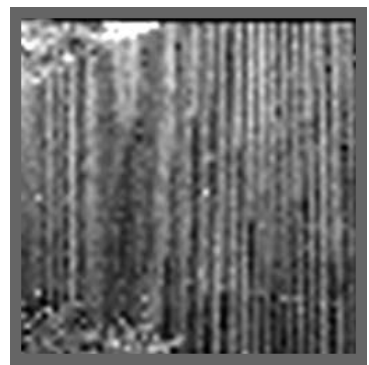

F. Cubic B-spline

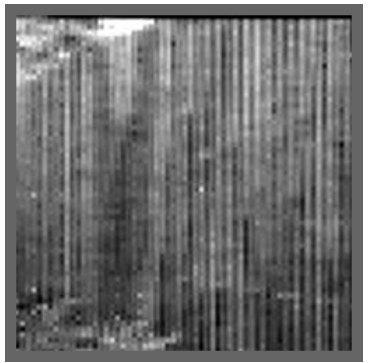

C. 2D-5PCC

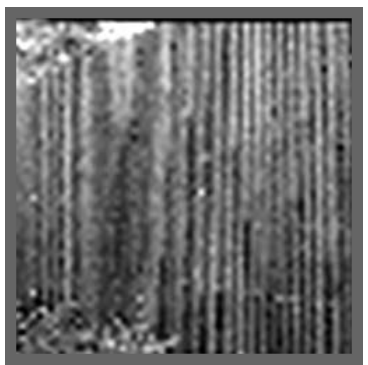

G. Cubic o-Moms

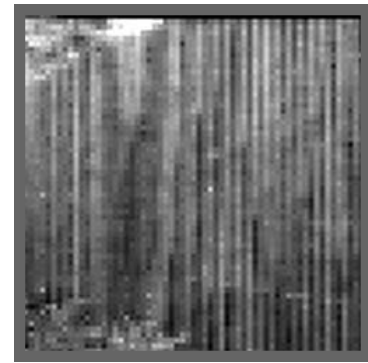

D. 2D-3PCC

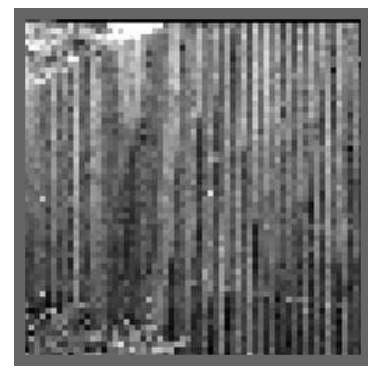

H. Nearest neighbor

Fig. 3. A $256 \times 256$ fence scene downsampled to $64 \times 64$, then interpolated to $256 \times 256$ by various algorithms.

Table 1. MSE results for the fence image.

\begin{tabular}{l||c|c|c}
\hline \multicolumn{1}{l||}{} & \multicolumn{3}{c}{ Mean Square Error } \\
Method & $128 \times 128$ & $64 \times 64$ & $32 \times 32$ \\
\hline Wiener & 0.112 & 0.225 & 0.295 \\
2D-5PCC & 0.169 & 0.342 & 0.471 \\
2D-3PCC & 0.170 & 0.352 & 0.506 \\
1D-1PCC & 0.182 & 0.404 & 0.554 \\
Cubic B-spline & 0.196 & 0.431 & 0.576 \\
Cubic o-Moms & 0.204 & 0.446 & 0.591 \\
Nearest neighbor & 0.185 & 0.453 & 0.612 \\
\hline
\end{tabular}

defined on $[-2,2] \times[-2,2]$ and constrained for continuity and smoothness. The closed-form solution for the optimal parameters is derived with respect to the power spectrum of an ensemble of scenes. Experimental results indicate that 2D-5PCC outperforms traditional methods in quantitative MSE and visual quality.

\section{REFERENCES}

[1] Erik Meijering, "A chronology of interpolation: From ancient astronomy to modern signal and image processing," Proceedings of the IEEE, vol. 90, no. 3, pp. 319-342, 2002.

[2] Samuel S. Rifman, "Digital rectification of ERTS multispectral imagery," in Proc. Symp. Significant Results Obtained from ERTS-1. NASA SP-327, 1973, vol. I Sec. B, pp. 11311142.

[3] Robert G. Keys, "Cubic convolution interpolation for digital image processing," IEEE Transactions on Acoustics, Speech, and Signal Processing, vol. 29, no. 6, pp. 1153-1160, 1981.

[4] Stephen K. Park and Robert A. Schowengerdt, "Image re- construction by parametric cubic convolution," Computer Vision, Graphics, and Image Processing, vol. 23, pp. 258272, 1983.

[5] Stephen E. Reichenbach and Frank Geng, "Improved cubic convolution for two-dimensional image reconstruction," in Nuclear Science Symposium, Medical Imaging Conference. IEEE, 2001, pp. 1775-1778.

[6] Carl L. Fales, Friedrich O. Huck, Judith A. McCormick, and Stephen K. Park, "Wiener restoration of sampled image data: End-to-end analysis," Journal of the Optical Society of America A, vol. 5, no. 3, pp. 300-314, 1988.

[7] A. Weber, "USC-SIPI Image Database, University of Southern California, sipi.usc.edu/services/database," 2004.

[8] M. Unser, "Splines: a perfect fit for signal and image processing," IEEE Signal Processing Magazine, vol. 16, no. 6, pp. 22-38, 1999.

[9] Thierry Blu, Philippe Thévenaz, and Michael Unser, "MOMS: maximal-order interpolation of minimal support," IEEE Transactions on Image Processing, vol. 10, no. 7, pp. 1069-1080, 2001.

[10] Philippe Thévenaz, Thierry Blu, and Michael Unser, "Interpolation revisited," IEEE Transactions on Medical Imaging, vol. 19, no. 7, pp. 739-758, 2000. 